\title{
Perspectiva de la dermatología y COVID-19
}

\author{
Natalia Calvache'; Juan Carlos Jaimes²; Paula Candelo3; Christian Prada4; Yurani \\ Pineda $^{5}$; Erika Peña ${ }^{6}$; Eduardo Fierro ${ }^{7}$
}

\section{RESUMEN}

La evidencia científica sobre manifestaciones cutáneas producidas por el nuevo coronavirus SARS-CoV-2 (COVID19) cada vez es mayor. La exacerbación de enfermedades cutáneas preexistentes y las lesiones por uso prolongado de elementos de protección personal son emergentes. Los especialistas en dermatología deben conocerlas, orientar en su prevención y tratamiento.

En este artículo se revisan las manifestaciones cutáneas en pacientes con COVID-19, las lesiones en piel que han surgido en trabajadores de la salud, la perspectiva de la teledermatología como herramienta para combatir la pandemia, algunas consideraciones especiales y protección de la piel y membranas mucosas.

PALABRAS CLAVE: infecciones por coronavirus; manifestaciones cutáneas; pandemias; personal de salud; dermatología; teledermatología.

1. Residente de Dermatología, Universidad Libre de Cali, Colombia. Grupo de Investigación en Dermatología (GRINDERM), Universidad Libre de Cali, Colombia. ORCID http://orcid.org/oooo-ooo1-8626-854X

2. Residente de Dermatología, Universidad Libre de Cali, Colombia. Grupo de Investigación en Dermatología (GRINDERM), Universidad Libre de Cali, Colombia. ORCID http://orcid.org/oooo-ooo2-5370-869X

3. Residente de Dermatología, Universidad Libre de Cali, Colombia. Grupo de Investigación en Dermatología (GRINDERM), Universidad Libre de Cali, Colombia. ORCID http://orcid.org/oooo-ooo2-4142-469X

4. Residente de Dermatología, Universidad Libre de Cali, Colombia. Grupo de Investigación en Dermatología (GRINDERM), Universidad Libre de Cali, Colombia. ORCID http://orcid.org/oooo-0001-7805-3741

5. Residente de Dermatología, Universidad Libre de Cali, Colombia. Grupo de Investigación en Dermatología (GRINDERM), Universidad Libre de Cali, Colombia. ORCID http://orcid.org/oooo-ooo2-2519-8610

6. Residente de Dermatología, Universidad Libre de Cali, Colombia. Grupo de Investigación en Dermatología (GRINDERM), Universidad Libre de Cali, Colombia. ORCID http://orcid.org/oooo-ooo1-9699-8140

7. Grupo de Investigación en Dermatología (GRINDERM), Universidad Libre de Cali, Colombia. Dermatólogo-oncólogo; Docente, Universidad Libre de Cali, Colombia. ORCID http://orcid.org/000o-0003-4910-5607

Correspondencia: Natalia Calvache; email: nataliacalvachernandez@gmail.com, nata_2794@hotmail.com

Recibido: 24/04/20; aceptado: 03/05/20

Cómo citar: Calvache N, Jaimes JJ, Candelo P, Prada C, Pineda Y, Peña E, Fierro E. Perspectiva de la dermatología y COVID-19. Rev Asoc Colomb Dermatol. 2020;28(1):17-26. DOI: https://doi.org/10.29176/2590843X.1487

Financiación: ninguna, conflictos de interés: ninguno 


\section{PERSPECTIVE OF DERMATOLOGY AND COVID-19}

\section{SUMMARY}

The scientific evidence of cutaneous manifestation produced by the new SARS-CoV-2 coronavirus (COVID19) is increasing. The exacerbation of pre-existing skin diseases and injuries due to prolonged use of personal protection elements are emerging. Dermatology specialists must know them and guide in prevention and treatment as well.

This article reviews the skin manifestations in patients with COVID-19, cutaneous lesions that have arisen in health workers, the perspective of teledermatology as a tool to fight against the pandemics, some special considerations, and protection of skin and mucous membranes.

KEY WORDS: Coronavirus infections; Skin manifestations; Pandemics; Health personnel; Dermatology; Teledermatology.

\section{INTRODUCCIÓN}

La Organización Mundial de la Salud (OMS) ha declarado la enfermedad por coronavirus 2019 (COVID-19) como una emergencia internacional de salud pública (1). El patógeno que causa la pandemia actual es un nuevo betacoronavirus, de origen zoonótico, denominado SARS-CoV-2 (severe acute respiratory syndrome coronavirus-2) (2). La transmisión ocurre persona-persona a través de gotas liberadas por boca o nariz y contacto con fómites infectados. El diagnóstico es con base en la clínica, laboratorios, imágenes y se confirma con el examen microbiológico ${ }^{(3)}$.

Es importante que el dermatólogo esté atento a documentarse sobre los nuevos retos de la pandemia, dado que a pesar de que el virus no es dermatotrópico, cada día hay mayor evidencia de lesiones cutáneas producidas por el nuevo coronavirus. Por otro lado, es importante resaltar que otras condiciones de la piel han emergido en este tiempo, resultado del uso permanente de equipos de protección personal (EPP), desinfectantes, lavado de manos más frecuente, así como exacerbaciones de enfermedades preexistentes, reacciones alérgicas, psicodermatosis y disminución de la vitamina D por el aislamiento prolongado, que requieren apoyo del especialista en piel.

Los dermatólogos deben asumir responsabilidades durante la pandemia, tales como proveer conocimiento sobre el cuidado de la piel y las mucosas al personal de salud y a la comunidad, informar sobre prevención y manejo de lesiones por uso de elementos de bioseguridad, aclarar cuáles enfermedades dermatológicas requieren o no tratamiento inmediato, emplear la teledermatología como herramienta de apoyo, sin dejar de lado la observación y el reporte de lesiones cutáneas tempranas y tardías en pacientes con COVID-19.

Esta revisión pretende recopilar la información disponible a la fecha acerca de la dermatología en la pandemia, con el objetivo de identificar situaciones actuales y la forma en la que se puede contribuir desde la perspectiva del dermatólogo.

\section{MANIFESTACIONES DERMATOLÓGICAS EN LOS PACIENTES CON COVID-19}

Existen pocos reportes sobre las manifestaciones cutáneas de COVID-19. Parece que la mayoría de las lesiones son brotes similares a los encontrados en otras infecciones virales y en las toxicodermias. Las evidencias son muy limitadas; en la literatura disponible hay pocas fotografías de las lesiones en piel, debido a las restricciones del uso de dispositivos en los cuartos de hospitalización durante la pandemia. Faltan estudios histopatológicos e incluso mayor reporte de pruebas confirmatorias de infección por SARS-CoV-2 por medio de reacción en cadena de la polimerasa de una muestra del tracto respiratorio superior.

Zhang y colaboradores reportaron las características clínicas de 140 pacientes infectados en Wuhan, China. Mencionaron que el 1,4\% ${ }^{(2)}$ presentaba urticaria crónica y el $11,4 \%{ }^{(4)}$ tenía historia de hipersensibilidad a drogas como comorbilidad asociada ${ }^{(5)}$.

Recalcati describió las manifestaciones dermatológicas en 88 pacientes con COVID-19, que fueron atendidos en 
un hospital italiano y sin consumo de medicamentos nuevos en los últimos 15 días ${ }^{(6)}$. El 20,4\%, correspondiente a 18 pacientes, desarrolló manifestaciones en piel, 8 al inicio de la hospitalización y 10 al egreso. Las lesiones fueron brote eritematoso (14 pacientes), urticaria generalizada (3 pacientes) y erupción variceliforme (1 paciente). El tronco fue la región más comprometida. El prurito fue poco frecuente o ausente y, al parecer, no había correlación con la gravedad de la enfermedad. Es importante considerar que el no consumo de medicamentos en las últimas dos semanas no excluye la posibilidad de toxicodermia, dado que medicamentos como los antimaláricos pueden presentar lesiones luego de más de dos semanas de su uso.

Otros estudios soportan el brote como manifestación de la infección. Hoenig y colaboradores describieron a un paciente joven con brote facial eritematoedematoso en la región malar, asociado a síntomas respiratorios y contacto con pacientes con COVID-19, como posible manifestación de la infección ${ }^{(7)}$. En un caso de Tailandia, reportaron un paciente que debutó con brote, petequias y trombocitopenia; inicialmente se consideró el diagnóstico de dengue y luego confirmaron infección por COVID-19 ${ }^{(8)}$. Un estudio que incluyó 1099 pacientes chinos con infección por SARS-CoV-2 reportó que $2(0,2 \%)$ presentaron brote como signo de infección ${ }^{(3)}$.

Marzano y colaboradores publicaron una serie de casos de 22 pacientes con exantema papulovesicular variceliforme como manifestación específica de COVID-19. En estos, las lesiones eran dispersas, con predominio de vesículas en el tronco y algunos en las extremidades. No hubo compromiso facial ni de mucosas ${ }^{(9)}$.

Algunos autores han postulado otras lesiones, tales como livedo reticular transitorio, púrpuras, petequias y acrocianosis de leve a moderada, como resultado de posibles eventos microembólicos del SARS-CoV-2. Estos hallazgos fueron reportados en 2 pacientes con COVID-19 no grave en Estados Unidos ${ }^{(10)}$. Otro estudio, publicado por Zhang y colaboradores, reunió 7 pacientes con neumonía crítica por COVID-19 y lesiones acroisquémicas. Los autores plantearon que esto era debido a un posible estado de hipercoagulación de esta presentación clínica ${ }^{(11)}$.

Con respecto a las manifestaciones dermatológicas en los niños, hay un reporte de caso de un paciente de 13 años con lesiones acroisquémicas. Inició con aparición de placas eritematosas y violáceas en los dedos de los pies y la superficie plantar, que evolucionaron a un aspecto purpúrico con costra negruzca, no dolorosas. Se acompañaron de síntomas sistémicos y contacto epidemiológico con la madre y la hermana con fiebre, tos y disnea. A pesar de la alta sospecha de COVID-19 y el antecedente de contacto epidemiológico, no fue posible confirmarlo con la prueba ${ }^{(12)}$.

Estos autores refieren que existen al menos una docena de casos de adolescentes y niños en Italia con estas mismas manifestaciones acrales, caracterizadas por una evolución subsecuente: inicialmente eritematovioláceas, luego ampollosas y con costra negra al final, además de historia familiar de COVID-19 ${ }^{(12)}$.

La letalidad de la COVID-19 es rara antes de la pubertad (<10 años) y la vulnerabilidad grave en hombres ha sido constantemente reportada ${ }^{(13)}$. Se ha considerado que la actividad del receptor de andrógenos y polimorfismos genéticos en el receptor convertidor de angiotensina 2 posiblemente intervengan en la transcripción de los genes correlacionados con el incremento de la carga viral y la gravedad del compromiso pulmonar. Signos de hiperandrogenismo pudieran ser esperados en estos pacientes ${ }^{(14)}$.

Ante la heterogeneidad de descripciones clínicas encontradas a nivel cutáneo en los pacientes con COVID-19, la Sociedad Española de Dermatología, en modalidad de consenso, definió la presencia de 5 patrones morfológicos en 375 pacientes con la infección; además, mostró su asociación con variables demográficas y gravedad (tabla 1) ${ }^{(15)}$.

\section{Histopatología de la COVID-19 y} piel

La elevada capacidad de contagio ha dificultado el conocimiento histopatológico de la infección por COVID-19 en piel. La información limitada ha sido producto de las indiscutibles restricciones de contacto y el carácter no esencial del estudio de la piel para el diagnóstico del coronavirus.

Un estudio en donde se publicó el resultado de una biopsia de piel de un brote por COVID-19 sugirió hallazgos similares a los encontrados en exantemas virales y dermatitis inducidas por fármacos. Se encontró infiltrado linfocítico perivascular superficial leve y vasos muy dilatados en la dermis papilar y media ${ }^{(16)}$. Estudios en cadáveres no encontraron evidencia de infección por coronavirus en piel (4). Estas 
Tabla 1. Características clínicas de pacientes con COVID-19 ${ }^{(15)}$.

\begin{tabular}{lccccc} 
& Pseudosabañón* & Vesicular & Urticarial & Maculopapular & Livedo/necrosis \\
\hline $\mathrm{n}(\%)$ & $71(19)$ & $34(9)$ & $73(19)$ & $176(47)$ & $21(6)$ \\
$\begin{array}{l}\text { Mujeres, } \mathrm{n}(\%) \\
\begin{array}{l}\text { Edad, promedio } \\
\text { (desviación }\end{array}\end{array}$ & $48(68)$ & $19(56)$ & $47(64)$ & $98(56)$ & $10(48)$ \\
$\begin{array}{l}\text { estándar) } \\
\text { Hospitalización, } \\
\mathrm{n}(\%)\end{array}$ & $32,5(21,8)$ & $45,6(20)$ & $48,7(19,9)$ & $55,3(20,2)$ & $63,1(17,3)$ \\
$\begin{array}{l}\text { UCl o ventilación } \\
\text { mecánica no } \\
\text { invasiva, } n \text { (\%) }\end{array}$ & $9(13)$ & $11(32)$ & $32(44)$ & $107(61)$ & $18(86)$
\end{tabular}

UCI: unidad de cuidados intensivos.

*Sabañón, en inglés chilblain.

características dan cimiento a la hipótesis de posible diseminación hematógena de la COVID-19.

\section{MANIFESTACIONES DERMATOLÓGICAS DE LOS TRABAJADORES DE LA SALUD QUE ATIENDEN PACIENTES CON COVID-19}

Los nuevos retos que enfrentan los trabajadores de la salud para combatir la pandemia han afectado su salud física y mental ${ }^{\left({ }^{17}\right)}$. El efecto de la hiperhidratación con los elementos de protección personal, la fricción, la ruptura de la barrera epidérmica y las reacciones de contacto a productos químicos para higiene de manos son los causantes de aumentar la incidencia de complicaciones cutáneas o de agravar las condiciones dermatológicas existentes ${ }^{(18,19)}$. La prevalencia de reacciones adversas en la piel durante la pandemia ha sido reportada entre $74,5 \%$ y $97 \%$, según estudios con base en encuestas al personal de salud en China ${ }^{(20,21)}$. Los sitios más afectados fueron las manos, las mejillas, la frente y el puente nasal en ambos estudios.

Con respecto a los síntomas, la xerosis fue el más frecuente $(70,3 \%)$, seguido de sensibilidad (56\%), prurito
(52\%) y ardor/dolor (38\%). Entre los signos, la descamación fue lo más encontrado $(61,6 \%)$, seguido de eritema (49\%), maceración (39\%), fisuras (38\%) y pápulas $(32 \%)^{(21)}$.

El tiempo de exposición a elementos de protección personal es un factor de riesgo importante para el desarrollo de lesiones cutáneas. Uso de gafas por más de 6 horas (odds ratio [OR], 2,32; IC 95\%: 1,41-3,83; $p<0,01)$, uso de máscara N95 por más de 6 horas al día (OR, 2,02; IC: 1,35-3,01; $p<0,01$ ) e higiene de manos más de 10 veces al día (OR, 2,17; IC 95\%: 1,38-3,43; $p<0,01)^{(21)}$. Otras variables que parecen aumentar el riesgo de reacciones cutáneas son: sexo femenino (OR, 1,87; $p=0,038)$, trabajar en hospitales con epidemia más grave (OR, 2,41; $p=0,001)$ y trabajar en salas de hospitalización (OR, 2,44; $p=0,003)^{(20)}$.

\section{TELEDERMATOLOGÍA COMO UNA HERRAMIENTA CONTRA LA INFECCIÓN POR SARS-COV-2}

La pandemia por COVID-19 ocasionó el cierre de muchos servicios no esenciales, siendo una medida de emergencia para prevenir la propagación. Como consecuencia, se disminuyó la consulta externa dermatológica para casos urgentes, incluidos procedimientos 
quirúrgicos para tumores malignos invasivos o interconsultas de pacientes hospitalizados con EPP adecuado y un énfasis en el distanciamiento social ${ }^{(22)}$.

Como alternativa a esta situación, se ha implementado nuevas tecnologías que transformaron el aislamiento preventivo en una oportunidad para el desarrollo de plataformas e infraestructuras. La teledermatología se ha convertido en una posible solución, que ha hecho que los dermatólogos se capaciten para garantizar su presencia virtual y brindar tratamiento a los pacientes ${ }^{(23)}$.

En el contexto de esta emergencia de salud pública, se deben conocer algunas limitaciones que pueden presentarse, como la calidad del video y de las imágenes, la velocidad y el acceso a internet y el cumplimiento de los pacientes ${ }^{(24)}$. Pese a esto, es una medida de acceso que ha logrado acortar tiempos de atención, brindar tratamiento y direccionamiento oportuno.

Durante la teleconsulta en tiempos de pandemia, es importante explicar al paciente que es una medida de contingencia por la COVID-19. Además, se debe informar cómo funciona la atención mediante el uso de tecnologías, los beneficios, el alcance, el manejo de la privacidad y de los datos personales. Debe quedar constancia en la historia clínica de que se obtuvo consentimiento informado, que el paciente comprendió la información dada y aceptó ser atendido por esta modalidad $^{(25)}$.

\section{CONSIDERACIONES ESPECIALES EN DERMATOLOGÍA}

\section{Procedimientos dermatológicos (toma de biopsia, dermatoscopia y cirugía)}

Los procedimientos diagnósticos y terapéuticos, como la toma de biopsias, las resecciones quirúrgicas, la crioterapia o la dermatoscopia, pueden requerir contacto estrecho con el paciente (definido como acercamiento a 2 metros y más de 15 minutos de exposición) (26), lo cual aumenta el riesgo de contagio. Para la realización de los procedimientos dermatológicos se recomienda ${ }^{(27,28)}$ :

- Diferir procedimientos en todos los pacientes con signos/síntomas sugestivos de infección respiratoria.
- Registrar en la historia el uso de elementos de protección por parte del personal médico y del paciente en los casos que sea posible.

- La dermatoscopia y los procedimientos en sitios como manos, uñas, cara, ojos y mucosas deben ser realizados solo cuando se considere esencial.

- Usar máscaras N95 y careta facial durante los procedimientos realizados en la cara, que requieren escisión o reconstrucción prolongada, especialmente aquellos en nariz o labios.

- Exigir al paciente el uso de una máscara y retirarla solo si interfiere con el sitio a evaluar o intervenir.

- Lavarse/desinfectarse las manos antes de realizar la dermatoscopia o cualquier procedimiento.

- Realizar dermatoscopia sin contacto. Limpiar con un paño y alcohol isopropílico al 70\% por al menos 1 minuto.

- Limpiar, con toallitas con alcohol isopropílico al $70 \%$, la parte del cuerpo que debe explorarse.

- Lavarse las manos adecuadamente, tanto el dermatólogo como el paciente, luego de haber realizado cualquier procedimiento.

- Realizar la limpieza de las salas de procedimientos, camas, sillas, teclados, teléfonos, interruptores, manijas de luz, pomos de las puertas, entre otros, con desinfectante con acción antiviral después de atender cada paciente.

- Usar, en lo posible, suturas absorbibles para evitar el desplazamiento o una nueva consulta.

- Resolver preguntas o dudas postoperatorias idealmente por teleconsulta.

- Tener presente que un solo dermatólogo debe realizar la dermatoscopia y si hay interés o duda, se debería tomar registro fotográfico para ser compartido posteriormente.

- Realizar limpieza de las salas de procedimientos, camas, sillas, teclados, interruptores, pomos de puertas, entre otros, después de la atención de cada paciente.

\section{Pacientes dermatooncológicos}

Por efecto de la pandemia de la COVID-19, es posible que sea necesario tomar decisiones terapéuticas que puedan estar fuera de las guías y recomendaciones. Es necesario informar a los pacientes de estas variaciones y resolver las dudas que puedan surgir. Las conductas elegidas deben ir consignadas en la historia clínica y en el consentimiento informado ${ }^{(29)}$. El panel de expertos de la National Comprehensive Cancer Network (NCCN) sugiere las siguientes recomendaciones de tratamiento en cuanto a los distintos tipos de patología oncológica en piel ${ }^{\left({ }^{\circ}\right)}$ : 
- La extirpación del carcinoma de células de Merkel idealmente no debe diferirse durante la pandemia, ya que el retraso conduce a progresión de la enfermedad y metástasis. Se pueden considerar excepciones para tumores $<1 \mathrm{~cm}$ en ancianos frágiles.

- Las resecciones locales y las cirugías de Mohs ambulatorias se podrían ejecutar, difiriendo la realización del ganglio centinela si no hay disponibilidad de quirófanos.

- Se recomienda diferir la resección del carcinoma basocelular superficial por 6 meses, excepto si la morbilidad es significativa. Para todos los otros tipos de carcinoma basocelular, se puede diferir la cirugía de 3 a 6 meses ${ }^{(31)}$.

- Ante queratosis actínicas y carcinoma escamocelular in situ, se recomienda diferir las cirugías. El carcinoma escamocelular invasivo requiere de triaje de acuerdo con los factores pronósticos ${ }^{(32)}$.

- Ante la sospecha de melanoma cutáneo primario, intentar realizar la biopsia escisional. Diferir las resecciones quirúrgicas en melanomas cutáneos in situ y T1 hasta por 3 meses, incluso en aquellos con márgenes positivos en la biopsia.

- Las biopsias del ganglio centinela pueden retrasarse hasta 3 meses, a menos que se realice al mismo tiempo que la resección local amplia en el quirófano.

- Aplazar controles posquirúrgicos de melanomas localizados en pacientes asintomáticos por 3 a 6 meses.

- Las resecciones metastásicas (estadios III y IV) deben colocarse en espera, a menos que el paciente esté muy sintomático, en cuyo caso, una dosis única de radioterapia paliativa podría ser una opción.

- Cuando se requiera, la terapia adyuvante puede iniciarse hasta 12 semanas desde el momento de la resección quirúrgica definitiva del melanoma. No se ha demostrado que la terapia adyuvante mejore la supervivencia específica del melanoma y debe diferirse durante la pandemia en pacientes con $<50 \%$ de probabilidad de recaída de la enfermedad.

- Se desconoce cómo reaccionarán los pacientes con COVID-19 en tratamiento con inmunoterapia y los eventos adversos esperados relacionados con el sistema inmunitario, siendo posible que sean más graves que el tratamiento con esteroides.

- Para el melanoma en estadio IV, se recomienda un único agente anti-PD-1 sobre la combinación de ipilimumab/nivolumab, porque hay menos inflamación y posible exacerbación de COVID-19, menos necesidad de esteroides para contrarrestar los eventos adversos y menos necesidad de seguimiento para verificar la toxicidad.

- En pacientes con carcinoma basocelular en tratamiento con terapia dirigida (vismodegid/ sonidegib), no hay pautas que recomienden suspender el tratamiento. Un estudio reciente de una clínica en Italia sugiere el ajuste de dosis para prolongar la duración del tratamiento, cuando sea posible ${ }^{(33)}$.

En pacientes con linfomas, se pueden clasificar por riesgo potencial de inmunosupresión según la terapia que estén recibiendo ${ }^{(34)}$ :

- Riesgo bajo: retinoides tópicos, mecloretamina tópica, esteroides tópicos con o sin oclusión, imiquimod, fototerapia UVB de banda estrecha domiciliaria (NB-UVB), helioterapia, antibióticos orales, antipruriginosos orales, baños de vinagre o lejía diluida/baños y humectación agresiva.

- Riesgo intermedio: retinoides orales (bexaroteno, acitretín, isotretinoína), metotrexato, esteroides orales, vorinostat e interferones (alfa o gamma).

- Riesgo alto: pralatrexato, romidepsina, mogamulizumab, brentuximab, gemcitabina y otras quimioterapias. La radioterapia cutánea, la fotoféresis y la terapia UV ambulatoria son de alto riesgo debido a los desplazamientos que implican.

Las terapias de bajo riesgo pueden utilizarse en el hogar y deben continuarse. Las terapias de riesgo intermedio instauradas se pueden continuar, pero con ajuste de dosis. El inicio de estas terapias puede posponerse utilizando terapias puente de bajo riesgo a corto plazo. Las terapias de alto riesgo, además de sus riesgos inherentes, pueden requerir desplazamiento a una institución hospitalaria. Solo deben utilizarse en pacientes con enfermedad avanzada y mayor compromiso sistémico. Los regímenes de infusión pueden ajustarse para aumentar los intervalos de tratamiento.

\section{Medicamentos}

\section{inmunomoduladores y biológicos}

\section{en la pandemia}

La evidencia aún no es contundente sobre los beneficios o los riesgos de suspender estos medicamentos 
durante la pandemia ${ }^{\left({ }^{35}\right)}$. Se debe considerar que ante la interrupción de biológicos en psoriasis, es posible que se disminuya la respuesta al reintroducirlos o, incluso, que haya desarrollo de anticuerpos ${ }^{(36)}$. Se ha sugerido algunas estrategias con base en los escenarios clínicos; sin embargo, la decisión de modificación o suspensión de tratamiento debe ser evaluada con el paciente y de manera interdisciplinaria ${ }^{(37)}$ :

Tabla 2. Recomendaciones en protección de la piel y las membranas mucosas.

La higiene de manos debe cumplir con "dos antes y tres después". Incluye:
antes de tocar a un paciente, antes de realizar cualquier procedimiento
aséptico, después de una posible exposición a fluidos del paciente,
Medidas de protección de
las manos
paciente o los elementos que puedan estar contaminados. Se recomienda
limpiar las manos con un desinfectante a base de alcohol al $70 \%$ o con
agua y jabón ${ }^{(28,38)}$.

Medidas de protección facial relacionada con máscaras y gafas
No respirar por la boca en áreas contaminadas o semicontaminadas. Evitar que los labios entren en contacto con el lado contaminado de las mascarillas. No tocar los labios hasta la desinfección estricta de las manos; cerrar los labios con fuerza al lavar la cara y el pelo para evitar que agua contaminada ingrese a la cavidad bucal. Evitar la exposición de mucosa ocular y piel periocular mediante el uso de gafas. Para tratar de mitigar el impacto de puntos de presión y laceraciones secundarias por uso de mascarillas y gafas, se aconseja su uso en posiciones diferentes, y si es el caso, utilizar gorro por debajo ${ }^{(28)}$.

Limpiar la cavidad nasal antes de salir de una zona limpia, salas aisladas o al salir del trabajo. La limpieza nasal debe realizarse con solución salina o agua y limpiar con hisopo de algodón humedecido durante 3-5 rondas. En caso de contaminación de las mascarillas faciales con sangre o fluidos del paciente, o directa contaminación a la cavidad nasal, el consenso recomienda la esterilización de acuerdo con los requisitos de las normas generales para la desinfección de las mucosas ${ }^{(28)}$.

Lavar el pelo con agua previo a tomar la ducha. Mantener los ojos, la nariz y la boca alejados del agua contaminada. Si el pelo no está expuesto al ambiente contaminado, el champú es suficiente. Si existe contaminación con sangre del paciente o por fluidos corporales, desinfectar inmediatamente con etanol al $75 \%$ y luego limpiar con champú ${ }^{(28)}$.

El uso continuo del EPP condujo a una mayor producción de sudor corporal, disbacteriosis cutánea y daños en la barrera, con el consecuente riesgo de Protección relacionada con disminución del rendimiento del EPP. Se recomienda controlar las horas de el sudor corporal trabajo con el EPP, tomar duchas de agua fría y uso de detergentes con $\mathrm{pH}$ ácido o neutro una vez que el personal salga de las áreas contaminadas, e inmediata humectación corporal ${ }^{(28)}$.

EPP: equipo de protección personal. 
- Pacientes que no muestran síntomas, o con síntomas respiratorios leves y sin contacto cercano con pacientes con COVID-19: continuar terapia con biológicos.

- Pacientes que experimentan síntomas respiratorios moderados o graves (fiebre, tos y/o dificultad para respirar) sin contacto cercano con pacientes confirmados con COVID-19: interrupción preventiva transitoria de la terapia biológica hasta la remisión completa de los síntomas respiratorios y al menos 72 horas sin fiebre.

- Pacientes con síntomas respiratorios leves y antecedentes de contacto con pacientes con COVID-19: interrupción del tratamiento preventivo de la terapia biológica hasta confirmar la negatividad de COVID-19 por laboratorio.

- Pacientes con síntomas respiratorios moderados a graves y contacto cercano o antecedentes de contacto con pacientes con COVID-19 o con criterios clínicos/radiológicos consistentes con COVID-19: interrupción de la terapia biológica e ingreso inmediato al hospital.

\section{PROTECCIÓN DE LA PIEL Y LAS MEMBRANAS MUCOSAS (TABLA 2)}

\section{CONCLUSIONES}

Las manifestaciones de COVID-19 en piel son múltiples. Entre las más frecuentes, el brote eritematoso, la urticaria, la erupción variceliforme y las lesiones vasculares como petequias, acrocianosis y livedo reticular transitorio. El uso de elementos de protección personal prolongado puede generar complicaciones cutáneas, con mayor proporción en las manos y la cara. Durante la pandemia, en los servicios de dermatología se debe priorizar la atención de pacientes, tomar precauciones para la realización de procedimientos e implementar la teleconsulta. Las recomendaciones son susceptibles a cambios a medida que se tiene más información concerniente a la pandemia. Por último, los dermatólogos y residentes de dermatología deben difundir información sobre la protección de la piel y las membranas mucosas a los trabajadores de la salud y a la comunidad.

\section{REFERENCIAS}

1. World Health Organization. Coronavirus disease (COVID-19) pandemic [Internet]. Disponible en: https://www.who.int.

2. Gorbalenya AE, Baker SC, Baric RS, de Groot RJ, Drosten C, Gulyaeva AA, Haagmans BL, Lauber C, Leontovich AM, Neuman BW, Penzar D, Perlman, et al. Severe acute respiratory syn-

\section{Puntos clave}

- Cada día hay mayor evidencia de lesiones cutáneas producidas por COVID19.

- Se deben identificar y tratar lesiones asociadas a elementos de protección personal.

- La atención presencial en tiempos de pandemia debe ser selectiva, para procedimientos y tratamientos de enfermedades cutáneas de alto riesgo.

- La teledermatología disminuye exposición innecesaria de pacientes y personal de salud frente al virus. 
drome related coronavirus: The species and its viruses a statement of the Coronavirus Study Group. bioRxiv. 2020.02.07.937862; doi: https://doi.org/10.1101/2020.02.07.937862.

3. Guan W, Ni Z, Hu Y, Liang W, Ou C, He J, Liu L, Shan H, Lei C, Hui D, Du B, Li L, et al. Clinical Characteristics of Coronavirus Disease 2019 in China. N Engl J Med. 2020;382(18):1708-20. doi: 10.1056/NEJMoa2002032.

4. Yao X, Li T, He Z, Ping Y, Liu H, Yu S, Mou HM, Wang LH, Zhang HR, Fu WJ, Luo T, Liu F, et al. [A pathological report of three COVID-19 cases by minimally invasive autopsies]. Zhonghua Bing Li Xue Za Zhi. 2020;49(5):411-7. doi: 10.376o/cma.j.cn112151-20200312-00193.

5. Zhang JJ, Dong X, Cao YY, Yuan YD, Yang YB, Yan YQ, Akdis C, Gao YD. Clinical characteristics of 140 patients infected with SARS-CoV-2 in Wuhan, China [Internet]. Allergy. 2020. doi: 10.1111/all.14238.

6. Recalcati S. Cutaneous manifestations in COVID19: a first perspective. J Eur Acad Dermatol Venereol. 2020;34(5):e212-3. doi: $10.1111 / j d v .16387$.

7. Hoenig L, Pereira F. Rash as a Clinical Manifestation of COVID 19 Photographs of a Patient. Clin Dermatol. 2020. doi: 10.1016/j.clindermatol.2020.04.001.

8. Joob B, Wiwanitkit V. COVID-19 can present with a rash and be mistaken for dengue. J Am Acad Dermatol. 2020;82(5):e177. doi: 10.1016/j.jaad.2020.03.036.

9. Marzano AV, Genovese G, Fabbrocini G, Pigatto P, Monfrecola G, Piraccini BM, Veraldi S, Rubegni P, Cusini M, Caputo V, Rongioletti F, Berti E, et al. Varicella-like exanthem as a specific COVID-19-associated skin manifestation: multicenter case series of 22 patients. J Am Acad Dermatol. 2020;S0190-9622(20)30657-5. doi: 10.1016/j.jaad.2020.04.044.

10. Manalo IF, Smith MK, Cheeley J, Jacobs R. A Dermatologic Manifestation of COVID-19: Transient Livedo Reticularis. J Am Acad Dermatol. 2020;So190-9622(20)30558-2. doi: 10.1016/j.jaad.2020.04.018.

11. Zhang Y, Cao W, Xiao M, Li Y, Yang Y, Zhao J, Zhou X, Jiang W, Zhao YQ, Zhang SY, Li TS. [Clinical and coagulation characteristics of 7 patients with critical COVID-2019 pneumonia and acro-ischemia]. Zhonghua Xue Ye Xue Za Zhi. 2020;41(o):Eoo6. doi: 10.376o/cma.j.issn.0253-2727.2020.0006.

12. Mazzotta F, Troccoli T. Acute acro-ischemia in the child at the time of COVID-19. Eur J Pediat Dermatol. 2020;(Monday's case):3-5.

13. Shi Y, Yu X, Zhao H, Wang H, Zhao R, Sheng J. Host susceptibility to severe COVID-19 and establishment of a host risk score: Findings of 487 cases outside Wuhan. Crit Care. 2020;24(1):108. doi: 10.1186/s13054-020-2833-7.

14. Wambier CG, Goren A. Severe Acute Respiratory Syndrome Coronavirus 2 (SARS-CoV-2) infection is likely to be androgen mediated. J Am Acad Dermatol. 2020;So190-9622(20)30608-3. doi: 10.1016/j.jaad.2020.04.032.

15. Galvan Casas C, Catala A, Carretero Hernandez G, Rodriguez-Jimenez P, Fernandez Nieto D, Rodriguez-Villa Lario A, Navarro Fernandez I, RuizVillaverde R, Falkenhain D, Llamas Velasco M, García-Gavin J, Baniandrés O, et al. Classification of the cutaneous manifestations of COVID-19: a rapid prospective nationwide consensus study in Spain with 375 cases. Br J Dermatol. 2020. doi: 10.1111/bjd.19163.

16. Zengarini C, Orioni G, Cascavilla A, Horna Solera C, Fulgaro C, Misciali C, Patrizi A, Gaspari V. Histological pattern in Covid-19 induced viral rash. J Eur Acad Dermatol Venereol. 2020. doi: 10.1111/jdv.16569.

17. Chawla S. COVID-19: Challenges and opportunities for dermatology response. J Dermatolog Treat. 2020;31(4):326. doi: 10.1080/09546634.2020.1751040.

18. Elston DM. Occupational skin disease among healthcare workers during the Coronavirus (COVID-19) epidemic. J Am Acad Dermatol. 2020;82(5):1085-1086. doi: 10.1016/j.jaad.2020.03.012.

19. Darlenski R, Tsankov N. Covid-19 pandemic and the skin - What should dermatologists know? Clin Dermatol. 2020. doi: https://doi.org/10.1016/j.clindermatol.2020.03.012.

20. Lin P, Zhu S, Huang Y, Li L, Tao J, Lei T, Song J, Liu D, Chen L, Shi Y, Jiang S, Liu Q, et al. Adverse Skin Reactions Among Healthcare Workers During the Coronavirus Disease 2019 Outbreak: A Survey in Wuhan and Its Surrounding Regions. Br J Dermatol. 2020. doi: 10.1111/bjd.19089.

21. Lan J, Song Z, Miao X, Li H, Li Y, Dong L, Yang J, An X, Zhang Y, Yang L, Zhou N, Yang L, et al. Skin damage among healthcare workers managing coronavirus disease-2019. J Am Acad Dermatol. 2020;82(5):1215-6. doi: 10.1016/j.jaad.2020.03.014. 
22. Villani A, Scalvenzi M, Fabbrocini G. Teledermatology: a useful tool to fight COVID19. J Dermatolog Treat. 2020;31(4):325. doi: 10.1080/09546634.2020.1750557.

23. Chawla S. COVID-19: Challenges and opportunities for dermatology response. J Dermatolog Treat. 2020;31(4):326. doi: 10.1080/09546634.2020.1751040.

24. Lee I, Kovarik C, Tejasvi T, Pizarro M, Lipoff JB. Telehealth: Helping Your Patients and Practice Survive and Thrive During the COVID-19 Crisis with Rapid Quality Implementation. J Am Acad Dermatol. 2020;82(5):1213-4. doi: 10.1016/j.jaad.2020.03.052.

25. Resolución 2654 de 2019. Por la cual se establecen disposiciones para la telesalud y parámetros para la práctica de la telemedicina en el país. Ministerio de Salud y Protección Social de Colombia.

26. Ministerio de Salud y Protección Social. Lineamientos para la detección y manejo de casos de COVID-19 por los prestadores de servicios de salud en Colombia. Ministerio de Salud y Protección social; 2020. Disponible en: https://bit.ly/2zg242J.

27. Jakhar D, Kaur I, Kaul S. Art of performing dermoscopy during the times of coronavirus disease (COVID-19): simple change in approach can save the day! J Eur Acad Dermatol Venereol. 2020. doi: 10.1111/jdv.16412.

28. Yan Y, Chen H, Chen L, Cheng B, Diao P, Dong L, Gao X, Gu H, He L, Ji C, Jin H, Lai W, et al. Consensus of Chinese experts on protection of skin and mucous membrane barrier for healthcare workers fighting against coronavirus disease 2019. Dermatol Ther. 2020;e13310. doi: 10.1111/dth.13310.

29. Cuevas-López L, Ayala J, Velásquez-Jiménez O, Navarro-Alean J, González-Higuera L, Zurita N, Hernández-Restrepo JD, Herrera Chaparro J, Ortiz D, Zuluaga-Zuluaga M, Bonilla-Ardila G. Recomendaciones para el manejo de los pacientes quirúrgicos urgentes durante la pandemia COVID-19. Rev Colomb Cir. 2020;35:143-52. doi: 10.30944/20117582.619.

30. National Comprenhensive Cancer Network NCCN. Advisory Statement for NonMelanoma Skin Cancer Care During the COVID-19 Pandemic [Internet]. Disponible en: https://www.nccn.org/covid-19/.

31. Der Sarkissian SA, Kim L, Veness M, Yiasemides E, Sebaratnam DF. Recommendations on dermatologic surgery during the COVID-19 pandemic. J Am
Acad Dermatol. 2020;So190-9622(20)30610-1. doi: 10.1016/j.jaad.2020.04.034.

32. Skulsky S, O’Sullivan B, McArdleO, Leader M, Roche M, Conlon P, O`Neill JP. Review of high-risk features of cutaneous squamous cell carcinoma and discrepancies between the American Joint Committee on Cancer and NCCN Clinical Practice Guidelines In Oncology. Head Neck. 2017;39(3):578-94. doi: 10.1002/hed.24580.

33. Villani A, Fabbrocini G, Costa C, Scalvenzi M. Patients with advanced basal cell carcinomas in treatment with sonic hedgehog inhibitors during the coronavirus disease 2019 (COVID-19) period: Management and adherence to treatment. J Am Acad Dermatol. 2020;82(6):e205. doi: 10.1016/j.jaad.2020.03.057.

34. Zic J, Ai W, Akilov O, Carter J, Duvic M, Foss F, Girardi M, Gru AA, Kim E, Musiek A, Olsen EA, Schieke SM, et al. United States Cutaneous Lymphoma Consortium. Recommendations for Treatment of Cutaneous Lymphomas During the COVID-19 Pandemic. J Am Acad Dermatol. 2020;So190-9622(20)30662-9. doi: 10.1016/j.jaad.2020.04.049.

35. Price K, Frew J, Hsiao J, Shi V. COVID-19 and immunomodulator/immunosuppressantusein dermatology.JAm Acad Dermatol. 2020;82(5):e173-e175. doi: 10.1016/j.jaad.2020.03.046.

36. Lebwohl M, Rivera-Oyola R, Murrell DF. Should biologicsforpsoriasisbeinterruptedintheeraofCOVID19? J Am Acad Dermatol. 2020;82(5):1217-8. doi: 10.1016/j.jaad.2020.03.031.

37. Di Lernia V. Biologics for psoriasis during COVID-19 outbreak. J Am Acad Dermatol. 2020;82(6):e217-8. doi: 10.1016/j.jaad.2020.04.004.

38. World Health Organization. Infection prevention and control during health care when COVID-19 is suspected: interim guidance, 19 March 2020. Disponible en: https://bit.ly/2A2Ao1a. 